\title{
Harnessing Light: the study and an update
}

\section{Arthur Guenther}

Arthur H. Guenther, "Harnessing Light: the study and an update," Proc. SPIE 4588, Seventh International Conference on Education and Training in Optics and Photonics, (28 May 2002); doi: 10.1117/12.468677

SPIE Event: Education and Training in Optics and Photonics 2001, 2001, Singapore, Singapore 


\title{
"HARNESSING LIGHT"*, The Study and an Update
}

\author{
Arthur H. Guenther** \\ University of New Mexico \\ Albuquerque, New Mexico, USA
}

\begin{abstract}
The completion of the report "Harnessing Light: Optical Science and Engineering for the $21^{\text {st }}$ Century" by COSE (Committee on Optical Science and Engineering) by the National Academy in 1998 has had a profound effect on optics related activities and the recognition of optics as a most pervasive and enabling field of technology. After a brief summary of the report - an update on its principal recommendations and other significant U.S. and global activities will be highlighted.
\end{abstract}

\section{The Study}

\section{The Field of Optics}

We live in a world bathed in light. We see with light, plants draw energy from light, and light is at the core of technologies from computing to surgical techniques. The field of optics, the subject of this report, concerns harnessing light to perform useful tasks.

Light influences our lives today in new ways that we could never have imagined just a few decades ago. As we move into the next century, light will play an even more significant role, enabling a revolution in world fiber-optic communications, new modalities in the practice of medicine, a more effective national defense, exploration of the frontiers of science, and much more.

We are beginning to see the fruits of the scientific discoveries of the last three or four decades. The development of the laser in the 1960s produced light with a property never seen before on Earth: coherence. Coherent light can be directed, focused, and propagated in new ways that are impossible for incoherent light. This property of laser light has made possible fiber-optic communications, compact disks, laser surgery, and a host of other applications--in all, a multitrilliondollar worldwide market. Applications of incoherent light abound as well, including optical lithography systems for patterning computer chips, high-resolution microscopes, adaptive optics for Earth-based astronomy, infrared sensors for everything from remote controls to night-vision equipment, and new high-efficiency lighting sources.

Although optics is pervasive in modern life, its role is that of a technological enabler: It is essential, but typically it plays a supporting role in a larger system. Central issues for this field include the following:

How to support and strengthen a field such as optics whose value is primarily enabling; and how to ensure the future vitality of a field that lacks a recognized academic or disciplinary home.

\footnotetext{
* National Research Council Report on Optics in the 21st Century Issue 98-3 OPTICS-TECHPOLICY May 15, 1998

Committee on Optical Science and Engineering releases NRC report

"Harnessing Light: Optical Science and Engineering for the 21 st Century.

** President, International Commission for Optics
} 


\section{The Report}

This report is the product of an unprecedented effort to bring together all aspects of the field of optics in one assessment organized around national needs. The report reviews the field's status today, assesses the outlook for tomorrow, and considers what must be done to assure the field's future vitality.

\section{Optics and National Needs}

Because optics applications are everywhere, this report is selective rather than comprehensive. It highlights areas where breakthroughs are taking place, where rapid change is likely in the near future, and where national needs dictate special attention. The field is largely defined by what it enables; as a result, applications drove the structure of the study and the report, which is organized around seven major areas of national need: (1) information technology and telecommunications; (2) health care and the life sciences; (3) optical sensing, lighting, and energy; (4) optics in manufacturing; (5) national defense; (6) manufacturing of optical components and systems; and (7) optics research and education. Some issues connected with these areas are outlined here. Some specific recommendations may be found in the Overview. More detailed conclusions on each area will be found in the chapters dedicated to those areas.

\section{Information Technology and Telecommunications}

In information technology, progress during the past decade has been extraordinary. For example, optical fiber for communications is being installed worldwide at a rate of 1,000 meters every second, comparable to the speed of a Mach 2 aircraft. Just 10 years ago, only 10 percent of all transcontinental calls in the United States were carried over fiber-optic cables; today 90 percent are. Meeting the computing and communications needs of the next 10 to 20 years will require advances across a broad front: transmission, switching, data storage, and displays. Many capabilities will have to advance a hundredfold. Although institutions have access to this rapidly growing, high-speed global telecommunications network, the infrastructure is not yet in place to provide individual consumer access that fully exploits the power of the system.

\section{Health Care and the Life Sciences}

In medicine, optics is enabling a wide variety of new therapies, from laser heart surgery to the minimally invasive knee repairs made possible by arthroscopies containing optical imaging systems. Optical techniques are under investigation for noninvasive diagnostic and monitoring applications such as early detection of breast cancer and "needle less" glucose monitoring for people with diabetes. Optics is providing new biological research tools for visualization, measurement, analysis, and manipulation. In biotechnology, lasers have become essential in DNA sequencing systems. Optics is playing such an important role in the life sciences and medicine that organizations concerned with these disciplines need to recognize and adjust to these developments.

\section{Optical Sensing, Lighting, and Energy}

Advances in lighting sources and light distribution systems are poised to dramatically reduce the one-fifth of U.S. electricity consumption now devoted to lighting. Innovative optical sensors are augmenting human vision, showing details and revealing information never before seen: infrared cameras that provide satellite pictures of clouds and weather patterns; night-vision scopes for use by law enforcement agencies; infrared motion detectors for home security, real-time measurements of industrial emissions, on-line industrial process control, and global environmental monitoring. Highresolution digital cameras are about to revolutionize and computerize photography and printing, and improvements in photovoltaic cells may permit solar energy to provide up to half of world energy needs by the middle of the next century. These developments will affect energy and environmental concerns on a national scale.

\section{Optics in Manufacturing}

Optics has had a dramatic economic influence in manufacturing, particularly since the advent of reliable low-cost lasers and laser-imaging systems. Optical techniques have become crucial in such diverse industries as semiconductor manufacturing, construction, and chemical production. Every semiconductor chip mass-produced in the world today is manufactured using optical lithography. Just making the equipment for this business is a $\$ 1$ billion industry, and it ultimately enables a $\$ 200$ billion electronics business. Other applications include laser welding and sintering, laser model generation, laser repair of semiconductor displays, curing of epoxy resins, diagnostic probes for real-time monitoring and 
control of chemical processes, optical techniques for alignment and inspection, machine vision, metrology, and even laser guidance systems for building tunnels. Optics can play an important role in ensuring a healthy U.S. manufacturing enterprise.

\section{National Defense}

In national defense, optical technology has become ubiquitous, from low-cost components to complex and expensive systems, and has dramatically changed the way wars are fought. Sophisticated satellite surveillance systems are a keystone of intelligence gathering. Night-vision imagers and missile guidance units allow the U.S. armed forces to "own the night". Lasers are used for everything from targeting and range finding to navigation, and may lead to high-power directed-energy weapons. The Department of Defense has a significant stake in optics.

\section{Manufacturing of Optical Systems and Components}

As the impact of optics has increased, changes have become necessary in how optical components and systems are designed and made. The manufacture of mass-market optics is now dominated by companies in Asia, but some recent developments are enabling U.S. industry to recapture selected market segments. One example is the emergence of new classes of numerically controlled optical grinding and polishing machines. Another is a better understanding of the characteristics of optical materials, from glasses to polymers to metals, thus permitting broader use of these automated technologies. Advanced optical components cannot be considered commodity items, and even though they represent only a small fraction of the value of the optical systems they enable, their availability is essential for the success of new highlevel applications that rely on those systems. The U.S. optics industry is currently strongest in the design and manufacture of high-performance specialty products. A key U.S. strength is in optical design, which is being revolutionized by the development of fast and affordable ray-tracing software. The United States can preserve a presence in world markets for optical components and systems by focusing on areas where domestic capabilities are strong and by addressing the process by which international standards are set.

\section{Education and Research}

Underpinning the explosive growth of optics are investments in education and research. Research continues to lead to extraordinary discoveries. Although the field is growing rapidly and its impact is both pervasive and far-reaching, it remains a "multidiscipline" with components in many university departments and government programs. The presence of optics in these diverse programs reflects its can easily be missed in such a disaggregated enterprise. Educational and research organizations will need to pay close attention to ensure that the field develops in a healthy way that ensures continuing benefits to society.

\section{The Update, Results to Date}

Each of the principal areas already covered in the Study as indicated in the Executive Summary contained a major highlighted recommendation that resulted from an analysis of testimonies given at the workshops. Findings were established, leading to conclusions, which resulted in a series of recommendations. Let us synopsize these high level recommendations and any significant progress. The reader is encouraged to refer to the text, "Harnessing Light, Optical Science and Engineering for the $21^{\text {st }}$ Century" for further details.

For Information Technology and Telecommunications it called for Congress to "lead the ... rapid development and deployment of broad band fibers to the home ...." As a result optical fibers to the home deployment has been developing but not as fast as had been anticipated.

In the area of Health Care, Biology, and Biotechnology the recommendation was that the National Institutes of Health should realize the need for increased innovative optical communications. It is our understanding that the National Institutes of Health is considering an organizational entity to address this area.

In the area of Optical Sensing, Lighting and Energy the recommendation was to aggressively pursue the development of more efficient light source to reduce energy requirements for illumination by a factor of 2 . We are pleased to note that the development of solid state light sources is aggressively being pursued in this area and that already light emitting diodes (LED) are replacing incandescent sources in a number of applications leading to greatly reduced power requirements and maintenance costs because of their long life and reliability. 
In the area of Defense, the recommendation was for the Department of Defense to continue their investment in optics and photonics advances and that attention be given to low cost manufacturing of aspheric, diffractive, and conformal optics. Here we are also pleased to note the development of numerically controlled fabrication of aspheres and the continued exploitation of diffractive optics together with major progress in the development of conformal programs.

In the area of Optics in Industrial Manufacturing which called for the development of precision laser materials processing by the establishment of test facilities in a service center scenario. While there are several that have been established a major facility from the Fraunhofer Institute in Germany has been establish in the Detroit, Michigan area to service the auto industries.

In the area of the Manufacture of Optical Components and Systems the National Institute of Standards and Technology (NIST) was encouraged to become a leader in the area of international optical standards setting for the U.S. The need for this recommendation has already been recognized by NIST, which has established "an initiative to encourage the nation to meet the challenge by insuring the vitality of its optics infrastructure".

Finally, in the area of Research and Education the value of optics as an enabling multidisciplinary technology should lead multiple agencies to support optics as a crosscutting initiative. We are pleased to note there is already an organization to establish and create a National Optics Initiative.

Other activities in education are being aggressively pursued by numerous organizations related to growing a workforce suitable to staff the expected explosive growth of optics and photonics. Thus the last response to the top-level recommendations in the report.

\section{Additional Items}

Other significant events following upon the publication of the Harnessing Light report include

- The formation of a Coalition for Photonics and Optics by a variety of professional and trade organization including a number of optics clusters. Additionally several organizations within the optics and photonics education field are present as observers.

- The NRC report "Harnessing Light, Optical Science and Engineering for the $21^{\text {st }}$ Century" has been distributed to all countries and territories of the International Commission for Optics, some 45 globally.

- In addition, a run of over 10,000 reprints of the Executive Summary has been accomplished and distributed to raise the visibility and enhance the recognition of optics as a most important pervasive and enabling technology that can address national needs including economic and defense security.

- As a result of the study there has been a stimulation of global optics planning both regionally and within specific countries, i.e., Germany, Singapore, South America, Ireland, Korea, Switzerland, etc.

- Developing nations which can benefit from the growth of an infusion of optics related industries such as Latin America and Africa are being targeted through the ICO and related affiliations for added attention especially in education and training.

- In the field of education various curricula are being developed for technician training as well as baccalaureate and graduate programs. One major initiative is STEP (Scientific and Technical Education in Photonics) by the National Science Foundation.

- Major U.S. optics enabled initiatives have taken place. Most prominent are those in information technology and nanoscience.

- Numerous economic assessments relating to the impact of optics/photonics and their expected growth have also taken place throughout the world.

- Finally, in preparation for a national initiative in optics, a White House conference on the economic impact of optics and photonics is planned for the spring of 2002.

In conclusion, the future looks bring and pervasive for optics and photonics in the $21^{\text {st }}$ Century. 